\title{
Prospects in double beta decay searches
}

\author{
S. Pirro ${ }^{a}$ \\ INFN - Sezione di Milano and Università di Milano Bicocca, Piazza della Scienza 3, I-20126 Milano, Italy
}

Received: 4 July 2005 /

Published online: 14 February 2006 - C Società Italiana di Fisica / Springer-Verlag 2006

\begin{abstract}
Neutrinos have recently provided us with the first tangible evidence of phenomena beyond the reach of our theory of the laws of particle physics, the remarkably predictive "Standard Model". The positive observation of oscillations in atmospheric and in solar neutrinos gives new motivations for more sensitive searches. Unfortunately, the oscillation experiments can only provide data on the squared mass differences of the neutrino mass eigenstates, while the absolute scale can only be obtained from direct mass measurements, ${ }^{3} \mathrm{H}$ end point measurements for example or, in the case of Majorana neutrinos, more sensitively by neutrinoless double beta decay. In fact, recently published constraints on the mixing angles of the neutrino mixing matrix make a strong case that if neutrinos are Majorana particles, there are many scenarios in which next-generation double beta decay experiments should be able to observe the phenomenon, trying to disentangle the mass scale of the neutrinos. The interest for next-generation double beta decay experiments is growing, for if the mass scale is below $\sim 0.2 \mathrm{eV}$, double beta decay may be the only hope for measuring it.
\end{abstract}

PACS. 23.40.Bw Weak-interaction and lepton (including neutrino) aspects - 11.30.Fs Global symmetries (e.g., baryon number, lepton number) - 14.60.Pq Neutrino mass and mixing

\section{Introduction}

All physical phenomena observed up to now in experiments performed with or without accelerators proved repeated confirmations of the validity of the Standard Model (SM). They enabled, moreover, the measurement of the set of fundamental constants which appear in their theoretical formalism. Many problems are still open, stimulating extensions of such a model: the high number of free parameters appearing in the SM (the three coupling constants, the masses of the fermions, the CabibboKobayashi-Maskawa matrix), the "running" coupling constants, the missing explanation of the hierarchy of the masses of the fermions. Recently, furthermore, with the discovery of neutrino oscillation the number of parameters increases with the need of introducing the analogue of the CKM matrix for the flavour mixing of neutrinos.

Following the pattern of what was done for the weak and electromagnetic interaction, physicists are looking for a "Unified Theory" of strong and electroweak interactions, named Grand Unified Theory (GUT). Such a theory should include the SM, in the same way as the weak theory includes electromagnetism and the theory of weak interactions. The fundamental idea is based on the assumption

a e-mail: Stefano.Pirro@mib.infn.it; Present address: INFN - Laboratori Nazionali del Gran Sasso, S.S. 17 bis km 18+910, Assergi (AQ), Italy. of the existence of an energy scale $M_{\mathrm{GUT}}\left(\sim 10^{15} \mathrm{GeV}\right)$ at which the three coupling constants, i.e. weak, electromagnetic and strong, converge toward a single coupling constant, which corresponds to a single interaction; such an interaction is based on a single gauge group. Many GUTs have been proposed, differing mainly in the choice of the gauge group on which they base themselves. The common characteristics of almost all GUTs are the violation of the lepton and baryon number. In order to have lepton number violation, the neutrino must be a Majorana particle.

As pointed out very recently by the Members of the APS Multidivisional Neutrino Study [1], Double Beta Decay (DBD) searches will play the central role in neutrino physics of the next decade.

\section{Dirac neutrinos and Majorana neutrinos}

Charged particles are, obviously, not their own antiparticles, due to the fact that they have an electric charge. In the same way, neutral particles, such as the neutron or the $\mathrm{K}^{0}$, differ from their antiparticles since they have a baryon number. Neutrinos, however, could be equal to their own antiparticles: in this case the neutrino would be a Majorana particle. In the framework of the Standard Model, massive Dirac neutrinos consist of four different states: suppose the existence of a neutrino with negative helicity (left handed) $\nu_{L}$; if CPT theorem holds, then there will 


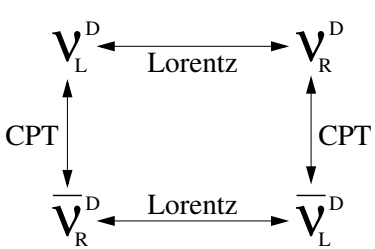

Dirac

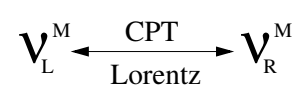

Majorana

Fig. 1. Transformations among neutrinos: Dirac neutrinos (left); Majorana neutrinos (right).

be the corresponding CPT-transformed state, i.e. an antineutrino with positive helicity (right handed), $\bar{\nu}_{R}$. Now, if the neutrino has a mass then there will exist a Lorentz boost that permits the helicity flip. Thus if the neutrino has a charge (lepton number) and a mass, it consists of four different states and is called a Dirac neutrino. If, on the contrary, the neutrino does not have a charge, only the two helicity states are defined; this is called a Majorana neutrino (see fig. 1). Due to the $V-A$ structure of the Standard Model the right-handed neutrinos are sterile, thus only two neutrinos are able to interact, as in the Majorana case. The difference is that in the Standard Model they interact due to their charge while in the Majorana case they interact due to their helicity.

From a theoretical point of view, the possibility that neutrinos are Majorana particles is particularly appealing. The fact that neutrinos and charged leptons, belonging to the same weak isodoublet, have an extremely different mass (at least a factor $10^{5}$ ) cannot be explained in the Dirac theory. Such an "anomaly" can be explained, without the insertion of an "ad hoc" symmetry, through the "see-saw" mechanism [2]: in the Majorana case, the mass of the neutrino naturally satisfies the relation $M_{\nu} M \approx$ $M_{q, \ell}^{2}$, where $M_{q, \ell}$ represents the mass of a lepton or a quark, and $M$ represents a mass scale.

Due to their tiny mass, the Dirac neutrinos in nature are produced, practically, always left handed, while anti-neutrinos are right handed. It is therefore impossible to discriminate whether they interact due to the lepton charge or due to their helicity. Different characteristics arise from the CPT transformation rules: it can be demonstrated [3] that if CPT is a conserved symmetry, then the Majorana neutrino cannot have an electric dipole or magnetic dipole moment. The magnetic moment for a Dirac neutrino can be evaluated [4] as $3.2 \cdot 10^{-19} m_{\nu} \mu_{B}$ ( $\mu_{B}$ is the Bohr magneton and $m_{\nu}$ is expressed in $\mathrm{eV}$ ). The present experimental limits are at least six orders of magnitude far away from the predicted value. There are other possibilities in order to search for lepton number non-conservation as in the case of pions and mesons decays [5]; the expected sensitivity, however, is very small compared to DBD. Thus the most favourable way to discriminate between Dirac and Majorana neutrino turns out to be the neutrinoless double beta decay $(0 \nu \mathrm{DBD})$.

After the discovery of neutrino oscillation, implying neutrino masses, the introduction of a mixing matrix between flavour eigenstates and mass eigenstates is straightforward. This matrix, analogous of the CKM matrix of the

quark sector, is the Pontecorvo-Maki-Nakagawa-Sakata (PMNS) matrix [6]. The flavour eigenstates can then be written as $\nu_{\alpha}=\sum_{i} U_{\alpha i} \nu_{i} . U_{\alpha i}$ are the elements of the PMNS matrix, and are related to the observable mixing angles in the base where the charged lepton masses are diagonal. For Majorana neutrinos there is an additional matrix that takes into account the fact that in this case there are 2 more complex phases. Using the Chau and Keung [7] parameterization of the PMNS matrix, we have:

$$
\begin{aligned}
& \left(\begin{array}{l}
\nu_{e} \\
\nu_{\mu} \\
\nu_{\tau}
\end{array}\right)=U V\left(\begin{array}{l}
\nu_{1} \\
\nu_{2} \\
\nu_{3}
\end{array}\right)= \\
& \left(\begin{array}{ccc}
c_{12} c_{13} & s_{12} c_{13} & s_{13} e^{-i \delta} \\
-s_{12} c_{23}-c_{12} s_{23} s_{13} e^{i \delta} & c_{12} c_{23}-s_{12} s_{23} s_{13} e^{i \delta} & s_{23} c_{13} \\
s_{12} s_{23}-c_{12} c_{23} s_{13} e^{i \delta} & -c_{12} s_{23}-s_{12} c_{23} s_{13} e^{i \delta} & c_{23} c_{13}
\end{array}\right) \\
& \times\left(\begin{array}{ccc}
1 & 0 & 0 \\
0 & e^{i \phi_{2} / 2} & 0 \\
0 & 0 & e^{i\left(\phi_{3} / 2+\delta\right)}
\end{array}\right)\left(\begin{array}{c}
\nu_{1} \\
\nu_{2} \\
\nu_{3}
\end{array}\right)
\end{aligned}
$$

where $c_{i j} \equiv \cos \theta_{i j}$ and $s_{i j} \equiv \sin \theta_{i j} ; \theta_{i j}$ are the mixing angles measured with the neutrino oscillations; $\delta$ is the Dirac CP phase. $V$ is a diagonal matrix containing the Majorana CP phases $\left(\phi_{2}\right.$ end $\left.\phi_{3}\right)$ that do not exist in the case of Dirac neutrinos and that, in any case, cancel in neutrino oscillations.

\section{Double beta decay}

The two-neutrino double beta decay mode $(2 \nu \mathrm{DBD})$ is expected to occur in the Standard Model as a secondorder effect of the well-known beta decay Hamiltonian, and it imposes no special requirements on the properties of the neutrino. It will occur irrespective of whether the neutrino is a Majorana or a Dirac particle and irrespective of whether it has a mass or not. The possible $2 \nu$ decay modes are

$$
\begin{array}{ll}
(A, Z) \rightarrow(A, Z+2)+2 e+2 \bar{\nu}_{e} & \beta \beta^{-} \\
(A, Z) \rightarrow(A, Z-2)+2 e^{+}+2 \nu_{e} & \beta \beta^{+} \\
(A, Z)+2 e^{-} \rightarrow(A, Z-2)+2 \nu_{e} & E C E C \\
(A, Z)+e^{-} \rightarrow(A, Z-2)+e^{+}+2 \nu_{e} & E C \beta^{+}
\end{array}
$$

Nuclear transitions accompanied by positron emission or electron capture processes are, however, characterized by poorer experimental sensitivities and will not be discussed in the following. The first process of eq. (2) represents the analogue of the single beta decay mediated by the weak current in which a $d$ quark transforms into an $u$ with the emission of an $e^{-}$and an $\bar{\nu}$; even if mediated by the same weak interaction, DBD is a second-order transition, resulting in an extremely slow decay rate, namely the slowest process ever observed in nature. Using simple dimensional arguments it is straightforward to demonstrate that, as a first approximation, the $2 \nu \mathrm{DBD}$ is $\sim 10^{21}$ times slower 


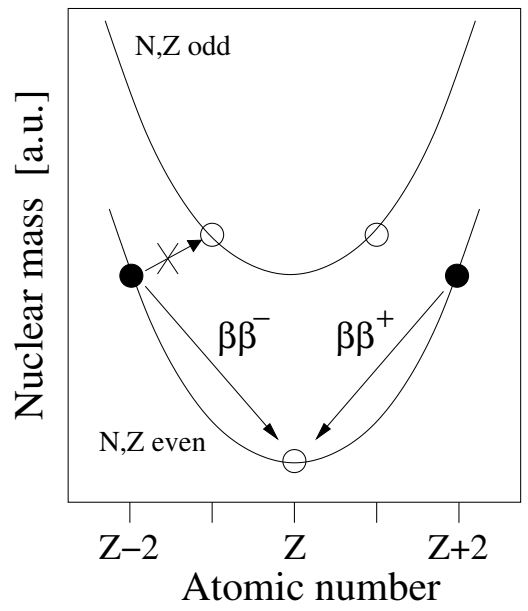

Fig. 2. Schematic picture of the atomic mass as a function of $Z$ for isobar multiplets with even $A$.
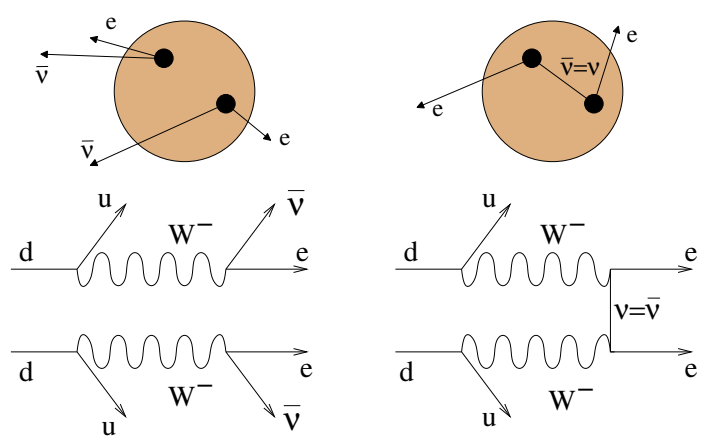

Fig. 3. Elementary scheme for $2 \nu \mathrm{DBD}$ (left) and $0 \nu \mathrm{DBD}$ (right).

with respect to the single beta decay, resulting in halflives of the order of $10^{18}-10^{22}$ years. The experimental observation of $2 \nu \mathrm{DBD}$ is therefore possible only if the single beta decay is prohibited by energy conservation or, at least, strongly hindered by small transition energy and/or by large change of angular momentum. This happens, fortunately, with several nuclei in nature and is due to the "pairing" interaction [8] (see fig. 2). The first "direct" observation of the $2 \nu \mathrm{DBD}$ was obtained in 1987 [9] and is now observed in more than ten nuclei $[10,11]$.

More interesting is the neutrinoless double beta decay $(0 \nu \mathrm{DBD})$, first proposed by Furry [12] in 1939. In this case there is the maximum lepton number violation $(\Delta L=2)$ and the decay is, therefore, not allowed by the Standard Model. The $0 \nu \mathrm{DBD}$ can occur only if two requirements are satisfied:

- the neutrino has to be a Majorana particle,

- the neutrino has to have a mass and/or the neutral current has to have a right-handed $(V+A)$ component.

The second condition is needed because of the helicity of the neutrino. Due to the $V-A$ nature of the weak interaction, the neutrino emitted in the first vertex (see fig. 3) is right handed, while in order to be absorbed in the second one, it needs to change its helicity. Thanks to the finite mass this is possible, with a probability $\propto m_{\nu} / E_{\nu}$;
Table 1. Theoretically evaluated $0 \nu \mathrm{DBD}$ half-lives (units of $10^{28}$ years) for $\left|\left\langle m_{\nu}\right\rangle\right|=10 \mathrm{meV}$. Only a few references are shown. The results of [13] are still considered by the authors as preliminary.

\begin{tabular}{lcccccc}
\hline Isotope & {$[14]$} & {$[15]$} & {$[16]$} & {$[13]$} & {$[17]$} & {$[18]$} \\
\hline${ }^{48} \mathrm{Ca}$ & 8.83 & - & - & - & 2.5 & - \\
${ }^{76} \mathrm{Ge}$ & 17.7 & 14.0 & 2.33 & 6.0 & 3.6 & 3.7 \\
${ }^{82} \mathrm{Se}$ & 2.4 & 5.6 & 0.6 & 1.8 & 1.5 & 0.81 \\
${ }^{100} \mathrm{Mo}$ & - & 1.0 & 1.28 & 3.5 & 3.9 & 0.65 \\
${ }^{116} \mathrm{Cd}$ & - & - & 0.48 & 2.4 & 4.7 & 0.39 \\
${ }^{130} \mathrm{Te}$ & 5.8 & 0.7 & 0.5 & 3.0 & 0.85 & 0.52 \\
${ }^{136} \mathrm{Xe}$ & 12.1 & 3.3 & 2.2 & 7.3 & 1.8 & 0.27 \\
${ }^{150} \mathrm{Nd}$ & - & - & 0.025 & $>0.3$ & - & - \\
\hline
\end{tabular}

from here it turns out that the amplitude of the decay is proportional to $m_{\nu}$. Disregarding more unconventional contributions (SUSY or left-right symmetric models), the $0 \nu \mathrm{DBD}$ rate is usually expressed as

$$
\left[T_{1 / 2}^{0 \nu}\right]^{-1}=G^{0 \nu}\left|M^{0 \nu}\right|^{2} \frac{\left|\left\langle m_{\nu}\right\rangle\right|^{2}}{m_{e}^{2}},
$$

where $G^{0 \nu}$ is the (exactly calculable) phase space integral $\propto Q_{\beta \beta}^{5}\left(Q_{\beta \beta}\right.$ represents the $Q$-value of the decay), $\left|M^{0 \nu}\right|^{2}$ is the specific nuclear matrix element of the nucleus undergoing the decay and $\left|\left\langle m_{\nu}\right\rangle\right|$ (effective electron neutrino mass, often called $\left.\left|m_{e e}\right|\right)$ is the neutrino relevant parameter measured in $0 \nu \mathrm{DBD}$. By using eq. (1) we have:

$$
\left.\left|\left\langle m_{\nu}\right\rangle\right| \equiv|| U_{11}\right|^{2} m_{1}+\left|U_{12}\right|^{2} m_{2} e^{i \phi_{2}}+\left|U_{13}\right|^{2} m_{3} e^{i \phi_{3}} \mid,
$$

where $e^{i \phi_{2}}$ and $e^{i \phi_{3}}$ are the Majorana CP phases $(= \pm 1$ in case of $\mathrm{CP}$ conservation), $m_{1,2,3}$ the mass eigenvalues and $U_{1 j}$ the matrix elements of the PMNS matrix. The presence of the $\phi_{k}$ phases implies that cancellations are, unfortunately, possible. Such cancellations are complete for a Dirac neutrino, since it is equivalent to two degenerate Majorana neutrinos with opposite CP phases. This stresses once more the fact that $0 \nu \mathrm{DBD}$ can occur only through the exchange of Majorana neutrinos.

From a Particle Physics point of view, $0 \nu \mathrm{DBD}$ represents a unique tool in order to measure the neutrino Majorana phases and to assess the absolute scale of the neutrino masses. As in evidence from eq. (3), the derivation of the crucial parameter $\left\langle m_{\nu}\right\rangle$ from the experimental results on $0 \nu \mathrm{DBD}$ lifetime requires a precise knowledge of the Nuclear Matrix Elements (NME) of the transition. Unfortunately, this is not an easy job, and a definite knowledge of NME values and uncertainties is still lacking in spite of the large attention attracted by this area of research. Many, often conflicting, evaluations are available in the literature and it is unfortunately not easy to judge their correctness or accuracy. Outstanding progress has been achieved over the last few years mainly due to the application of the QRPA method and its extensions. Renewed interest in Shell Model calculations has been boosted, on the other hand, by the fast development of computer technologies. Comparison with experimental $2 \nu$ DBD rates has often been suggested as a possible way out (direct test of 
Table 2. Summary of the present information on neutrino masses and mixings from oscillation data. For a recent review see [19].

\begin{tabular}{lcc}
\hline Oscillation parameter & Central value & $99 \%$ CL range \\
\hline Solar mass splitting & $\Delta m_{12}^{2}=(8.0 \pm 0.3) 10^{-5} \mathrm{eV}^{2}$ & $(7.2-8.9) 10^{-5} \mathrm{eV}^{2}$ \\
Atmospheric mass splitting & $\left|\Delta m_{23}^{2}\right|=(2.5 \pm 0.3) 10^{-3} \mathrm{eV}^{2}$ & $(1.7-3.3) 10^{-3} \mathrm{eV}^{2}$ \\
Solar mixing angle & $\tan ^{2} \theta_{12}=0.45 \pm 0.05$ & $30^{\circ}<\theta_{12}<38^{\circ}$ \\
Atmospheric mixing angle & $\sin ^{2} 2 \theta_{23}=1.02 \pm 0.04$ & $36^{\circ}<\theta_{23}<54^{\circ}$ \\
"CHOOZ" mixing angle & $\sin ^{2} 2 \theta_{13}=0 \pm 0.05$ & $\theta_{13}<10^{\circ}$ \\
\hline
\end{tabular}

the calculation method). The evaluation methods for the two decay modes show, however, relevant differences (e.g. the neutrino propagator), and the effectiveness of such a comparison is still controversial [13,20,21]. A popular but doubtful attitude consists in considering the spread of the different evaluations as an estimate of their uncertainties. In such a way one obtains a spread of about one order of magnitude in the expected half-lives (table 1), corresponding to a factor of $\sim 3$ in $\left\langle m_{\nu}\right\rangle$. It is clear that a big improvement in the calculation of NME or, at least, in the estimate of their uncertainties would be welcomed. New calculation methods should be pursued while insisting on the comparison with dedicated measurements coming from various areas of nuclear physics [22]. On the other hand, an experimental effort to investigate as many $\beta \beta$ emitters as possible should be addressed.

\section{Prediction on the Majorana mass}

The input parameters in order to figure out the possible mass pattern of the Majorana neutrinos are given by the neutrino oscillations. The most updated values $[23,24,25$, $26,27,28]$ are given in table 2 .

Given the two $\Delta m^{2}$ measured with the oscillations, and given the assumption of three neutrinos, the absolute mass scale is still missing. Nevertheless, three scenarios are possible, as shown in fig. 4 . The previously quoted values can be accommodated in the framework of three neutrinos mixing, which describes the three flavour neutrinos $\left(\nu_{e}\right.$, $\nu_{\mu}$ and $\left.\nu_{\tau}\right)$ as unitary linear combinations of the three massive neutrinos $\left(\nu_{1}, \nu_{2}\right.$ and $\left.\nu_{3}\right)$ having masses $m_{1}, m_{2}$, and $m_{3}$, respectively. The experimental measurements are compatible with three mass schemes:

1. Normal hierarchy: $m_{1}<m_{2}<m_{3}$, i.e. $\Delta m_{23}^{2}>0$,

$$
\Delta m_{12}^{2} \simeq \Delta m_{\text {sun }}^{2} \simeq m_{2}^{2}, \quad \Delta m_{23}^{2} \simeq\left|\Delta m_{\text {atm }}^{2}\right| \simeq m_{3}^{2}
$$

2. Inverted hierarchy: $m_{3}<m_{1}<m_{2}$, i.e. $\Delta m_{23}^{2}<0$,

$$
\Delta m_{12}^{2} \simeq \Delta m_{\mathrm{sun}}^{2}, \quad \Delta m_{23}^{2} \simeq-\left|\Delta m_{\mathrm{atm}}^{2}\right| \simeq-m_{1}^{2} .
$$

3. Degenerate case: the values of $\Delta m_{i j}^{2}$ are small when compared to each mass values. In this case the hierarchies are undistinguishable:

$$
\left|\Delta m_{i j}^{2}\right| \ll m_{1}^{2} \simeq m_{2}^{2} \simeq m_{3}^{2} .
$$

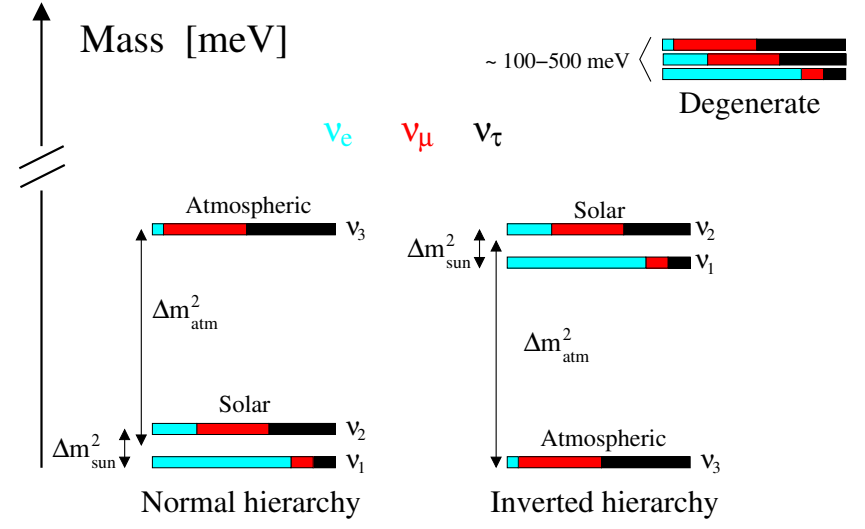

Fig. 4. Neutrino mass pattern based on the experimental relation $\Delta m_{\text {sun }}^{2} \ll \Delta m_{\text {atm }}^{2}$.

In order to extract the correct prediction, however, one has to use eq. (4); the steps are:

- use the matrix elements given by eq. (1) with the evaluated neutrino parameter from table 2 ,

- parameterize $m_{2}=\sqrt{m_{1}^{2}+\Delta m_{\text {sun }}^{2}}$ and

$$
m_{3}=\sqrt{m_{2}^{2}+\left|\Delta m_{\text {atm }}^{2}\right|} \text { for normal hierarchy, }
$$

- parametrize $m_{1}=\sqrt{m_{3}^{2}+\left|\Delta m_{\text {atm }}^{2}\right|}$ and

$$
m_{2}=\sqrt{m_{1}^{2}+\Delta m_{\mathrm{sun}}^{2}} \text { for inverted hierarchy. }
$$

With this procedure the effective Majorana mass can be written as a function of the lightest neutrino mass $\left(m_{\text {light }} \equiv m_{1}\right.$ for normal hierarchy, $m_{\text {light }} \equiv m_{3}$ for inverted hierarchy):

$$
\left|\left\langle m_{\nu}\right\rangle\right| \equiv\left|m_{e e}\right|=f\left(m_{\text {light }}, \phi_{1}, \phi_{2}, \text { observables }\right),
$$

where observables are all the experimental data from neutrino oscillations. The plot of $\left|\left\langle m_{\nu}\right\rangle\right| \equiv\left|m_{e e}\right|$ is shown in fig. 5. The two disfavoured regions are given by the present limits on DBD experiments (see table 3) and by cosmological (Large-Scale Structures and anisotropies in the Cosmic Microwave Background) bounds [29].

The DBD-Experiments developed up to now, often called First-Generation Experiments, were designed to explore only the degenerate mass scenario. The proposed Second-Generation Experiments are designed to explore the inverted hierarchy scenario, with an expected sensitivity on $\left|m_{e e}\right|$ of the order of $10-50 \mathrm{meV}$. 
Table 3. Best reported results on $0 \nu$ DBD processes. Limits are at $90 \%$ CL except when noted. The effective neutrino mass limits and ranges are those deduced by the authors $\left(\left\langle m_{\nu}\right\rangle\right)$ or according to table $1\left(\left\langle m_{\nu}^{\dagger}\right\rangle\right)$. Only $\ddagger$ nuclei are presently investigated by high-sensitivity experiments.

\begin{tabular}{lllllll}
\hline Isotope & $Q_{\beta \beta}(\mathrm{keV})$ & $i . a$. & $T_{1 / 2}^{2 \nu}(\mathrm{y})$ & $T_{1 / 2}^{0 \nu}(\mathrm{y})$ & $\left\langle m_{\nu}\right\rangle(\mathrm{eV})$ & $\left\langle m_{\nu}^{\dagger}\right\rangle(\mathrm{eV})$ \\
\hline${ }^{48} \mathrm{Ca}$ & 4271 & 0.187 & $(4.2 \pm 1.2) \times 10^{19}$ & $>9.5 \times 10^{21}(76 \%)[30]$ & $<8.3$ & $<30$ \\
${ }^{76} \mathrm{Ge}$ & 2039 & 7.8 & $(1.3 \pm 0.1) \times 10^{21}$ & $>1.9 \times 10^{25}[31,32]$ & $<0.35$ & $<0.35-0.96$ \\
${ }^{82} \mathrm{Se} \ddagger$ & 2995 & 9 & $(9.6 \pm 1.0) \times 10^{19}$ & $>1.6 \times 10^{25}[33,34]$ & $<0.38-1.05$ & $<0.38-1.05$ \\
${ }^{100} \mathrm{Mo} \ddagger$ & 3034 & 9.6 & $(7.11 \pm 0.54) \times 10^{18}$ & $>4.6 \times 10^{23}[35]$ & $<1.7-4.9$ & $<2.2-6.9$ \\
${ }^{116} \mathrm{Cd}$ & 2806 & 7.5 & $(2.9 \pm 0.4) \times 10^{19}$ & $>1.7 \times 10^{23}[36]$ & $<0.7-2.8$ & $<1.36-3.3$ \\
${ }^{130} \mathrm{Te} \ddagger$ & 2528 & 33.9 & $(6.1 \pm 1.4) \times 10^{20}$ & $>1.8 \times 10^{24}[37,38]$ & $<0.2-1.1$ & $<0.5-1.8$ \\
${ }^{136} \mathrm{Xe}$ & 2479 & 8.9 & $>1.6 \times 10^{22}$ & $>1.2 \times 10^{24}[39]$ & $<1.1-2.9$ & $<0.5-3.2$ \\
${ }^{150} \mathrm{Nd}$ & 3367 & 5.6 & $7.0_{-0.3}^{+11.8} \times 10^{18}$ & $>1.2 \times 10^{21}[40]$ & $<3$ & $<4.6-6.5$ \\
\hline
\end{tabular}

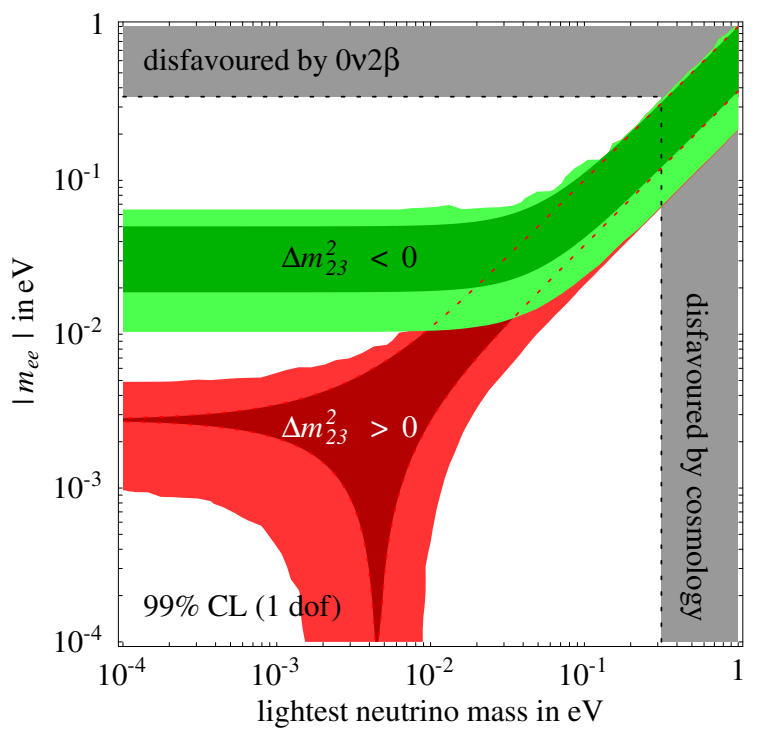

Fig. 5. 99\% CL range for $m_{e e}$ as function of the lightest neutrino mass. The darker regions show how the $m_{e e}$ range would shrink if the best-fit values of oscillation parameters were confirmed with negligible errors (in this case the spread is due only to the Majorana CP phases). Picture given by courtesy of the authors of [19].

\section{Experimental techniques}

The experimental signatures of the nuclear double beta decays are in principle very clear: in the case of the $0 \nu \mathrm{DBD}$, one should expect a peak (at the $Q_{\beta \beta}$ value) in the twoelectrons summed energy spectrum, whereas a continuous spectrum (with a well-defined shape) will feature the $2 \nu \mathrm{DBD}$.

In spite of such characteristic imprints, the rarity of the processes under consideration makes their identification very difficult. Such remotely probable signals have to be disentangled from a background due to natural radioactive decay chains, cosmogenic-induced activity, and man-made radioactivity, which deposit energy in the same region as the DBD, but at a faster rate. Consequently, the main task in $0 \nu \mathrm{DBD}$ searches is to diminish the background by using the state-of-the-art ultra-low background techniques and, hopefully, identifying the signal. Many are the experiments searching for DBD. They may be substantially subdivided into three categories:

- Geochemical determination of the total decay time through the measurement of the daughter nuclei $(A, Z+2)$ produced by the parent nuclei $(A, Z)$ in a sample of "old" geological rocks.

- Radiochemical measurement of the total decay time by the extraction of the radioactive daughter nuclei from the parent nuclei.

- Direct detection of the two electrons emitted in the transition.

The experiments belonging to the first two classes do not allow to distinguish among the two decay channels. They are, however, extremely sensitive to inclusive effects, since the so called "accumulation times" for the daughter isotope are very long. They gave, however, the first $i n$ direct prove [41] of the existence of the $2 \nu \mathrm{DBD}$, but are no longer pursued nowadays. Direct experiments are the most interesting ones because they allow to distinguish the various modes of the double beta decay. It is therefore possible to search for the decay without neutrinos, which represents the most interesting process. The nuclear detector capable of revealing the two electrons emitted by the DBD-Emitter should have some basic properties:

- High energy resolution, since a peak must be identified over a background.

- Low background, which requires the use of extremely radiopure materials: natural radioactivity (mainly ${ }^{232} \mathrm{Th}$ and ${ }^{238} \mathrm{U}$ chains) exhibits decay times of the order of $10^{9}$ years, extremely short if compared with the expected $0 \nu \mathrm{DBD}$ that should have a decay time larger than $10^{25}$ years. Furthermore it is absolutely necessary to operate the detector in underground laboratories in order to shield cosmic rays.

- Large source of DBD nuclei in order to have sensitivity to the decay time up to $10^{25}-10^{28}$ years.

- Event reconstruction methods, since the $0 \nu \mathrm{DBD}$ has a very characteristic decay with the two electrons that share the $Q_{\beta \beta}$ energy. Electron tracking can therefore help in rejecting background. 
Unfortunately there are up to now no detectors that can fulfill these four requirements at the same time. Two are, substantially, the experimental approaches:

- homogenous detectors (or active source detectors), whose mean feature is to have the active $\beta \beta$ source inside the detector material,

- non-homogeneous detectors (or passive source detectors), in which the source and the detector are distinct.

Various conventional counters have been used so far in DBD direct searches: solid state devices (germanium spectrometers and silicon detector stacks), gas counters (time projection chambers, ionization and multiwire drift chambers) and scintillators (crystal scintillators and stacks of plastic scintillators). Techniques based on the use of low temperature true calorimeters have been, on the other hand, proposed and developed in order to improve the experimental sensitivity and enlarge the choice of suitable candidates for DBD searches investigable with an active source approach.

A common feature of all DBD experiments has been the constant work against backgrounds caused mainly by environmental radioactivity, cosmic radiation and residual radioactive contaminations of the detector setup elements. The further suppression of such backgrounds will be the actual challenge for future projects whose main goal will be to maximize the $0 \nu \mathrm{DBD}$ rate while minimizing background contributions.

In order to compare different experiments and in order to point out the advantages and the disadvantages of the two different detecting techniques, it is convenient to introduce a very important parameter, called "sensitivity", denoted by $F$. It is defined as the process halflife corresponding to the maximum number of signals $(n)$ that could be hidden by the background fluctuations, at a given statistical CL. Let $t$ be the measurement time, $N_{\beta \beta}$ be the number of atom candidates for $\beta \beta$ decay present in the source, $B$ (expressed in number of counts per unit energy per unit time) the background counting rate in the energy region where the decay peak is expected and $\Delta E$ the FWHM energy resolution. The expected number of background counts in an energy interval equal to the FWHM energy resolution centered at the transition energy can then be written as $N_{B}=B \Delta E t$. The sensitivity factor $F$ at $1 \sigma$ level $\left(n=\sqrt{N_{B}}\right)$ is defined as

$$
F \equiv T_{1 / 2}=\frac{\ln 2 N_{\beta \beta} t}{n}=\ln 2 N_{\beta \beta} \sqrt{\frac{t}{B \Delta E}}
$$

If the background (measured as counts per unit energy per unit mass per unit time) is proportional to the detector mass the above formula can be rewritten as

$$
F=\ln 2 \frac{i . a . \epsilon x}{A} N_{A} \sqrt{\frac{M t}{B \Delta E}} \quad(68 \% \mathrm{CL}),
$$

where $A$ is the compound molecular mass, $x$ the number of $\beta \beta$ atoms per molecule, i.a. their isotopic abundance, $M$ the source mass, $N_{A}$ the Avogadro's number and $\epsilon$ the efficiency of the detector. In addition to its simplicity, eq. (10) has the advantage of emphasizing the role of the essential experimental parameters: mass, measuring time, isotopic abundance, background level, energy resolution and detection efficiency.

As far as the active source experiments are concerned, they can have detection efficiencies of the order of $\sim 90 \%$, and energy resolution of the order of $\sim 0.2 \% \mathrm{FWHM}$ (for Ge diodes and bolometers), while the background (mainly arising from the surrounding setup) cannot be easily rejected. As far as the passive source experiments are concerned, they are mostly performed with gas detectors (TPC, DC) in which the source is introduced into the volume of the detector as very thin sheets (of about $50 \mu \mathrm{m})$, to reduce the energy loss of the electrons emitted in the decay. The detection efficiency associated with this kind of measure is of the order of $30 \%$. The great advantage of these experiments lies in the reduction of the background: the clear trace, which is peculiar in a drift chamber, for a 2 electrons event, guarantees a very good capability of background discrimination. The energy resolution, on the other hand, cannot be as good as $\sim 7-10 \%$ FWHM. As will be shown in sect. 7 the energy resolution plays an extremely crucial role in the second-generation experiments due to the fact that the $2 \nu \mathrm{DBD}$ close to the endpoint will result in an unavoidable/unrejectable source of background for the $0 \nu \mathrm{DBD}$ mode.

\section{First-generation experiments}

Impressive progress has been obtained during the last years in improving $0 \nu \mathrm{DBD}$ half-life limits for several isotopes and in systematically cataloguing $2 \nu \mathrm{DBD}$ rates (table 3 ). Although $2 \nu \mathrm{DBD}$ results are in some cases inconsistent, the effort to cover as many $\beta \beta$ nuclei as possible thus allowing a direct check for $2 \nu \mathrm{DBD}$ NME is evident. Optimal $0 \nu$ DBD sensitivities have been reached in a series of experiments based on the active source approach. In particular, the best limit on $0 \nu \mathrm{DBD}$ comes from the Heidelberg-Moscow (HM) experiment [31] on ${ }^{76} \mathrm{Ge}$ even if similar results have been obtained also by the IGEX experiment [34] (table 3).

In both cases a large mass (several $\mathrm{kg}$ ) of isotopically enriched $(86 \%)$ germanium diodes, is installed deep underground under heavy shields for gamma and neutron environmental radiation. Extremely low background levels are then achieved thanks to a careful selection of the setup materials and further improved by the use of pulse shape discrimination (PSD) techniques. Both experiments quote similar background levels in the $0 \nu \mathrm{DBD}$ region of $\approx 0.2(\mathrm{c} / \mathrm{keV} \cdot \mathrm{kg} \cdot \mathrm{y})$ and $\approx 0.06(\mathrm{c} / \mathrm{keV} \cdot \mathrm{kg} \cdot \mathrm{y})$ before and after PSD. Taking into account the uncertainties in the NME calculations, such experiments indicate a limit of $0.3-1 \mathrm{eV}$ for $\left\langle m_{\nu}\right\rangle$.

As will be discussed later, new ideas to improve such a successful technique characterize many of the proposed future projects. However, given the NME calculation problem, more $\beta \beta$ emitters than allowed by the use of 
conventional detectors (e.g. ${ }^{76} \mathrm{Ge},{ }^{136} \mathrm{Xe},{ }^{116} \mathrm{Cd}$ ) should be investigated using the calorimetric approach. A solution to this problem, suggested [42] and developed [43] by the Milano group, is based on the use of low temperature calorimeters (bolometers). Besides providing very good energy resolutions they can in fact practically eliminate any constraint in the choice of the $\beta \beta$ emitter. Due to their very simple concept (a massive absorber in thermal contact with a suitable thermometer measuring the temperature increase following an energy deposition), they are in fact constrained only by the requirement of finding a compound allowing the growth of a diamagnetic and dielectric crystal. Extremely massive [44] detectors can then be built, by assembling large crystal arrays.

Thermal detectors have been pioneered by the Milano group for ${ }^{130} \mathrm{Te}$ (chosen, because of its favourable nuclear factor-of-merit and large natural isotopic abundance, from a large number of other successfully tested $\beta \beta$ emitters) in a series of constantly increasing mass experiments carried out at Laboratori Nazionali del Gran Sasso (LNGS), whose last extension, started in 2003, is the CUORICINO experiment [38]. Consisting of an array of $62 \mathrm{TeO}_{2}$ crystals totalling a mass of $40.7 \mathrm{~kg}$ and operating at a temperature of $\sim 8-9 \mathrm{mK}$, CUORICINO is characterized by a good energy resolution $(7-8 \mathrm{keV}$ on the average at the $0 \nu \mathrm{DBD}$ transition energy, $2528 \mathrm{keV}$ ) and a background level of $\sim 0.18(\mathrm{c} / \mathrm{keV} \cdot \mathrm{kg} \cdot \mathrm{y})$. The quoted limit of $1.8 \times 10^{24} \mathrm{y}$ on the ${ }^{130} \mathrm{Te} 0 \nu \mathrm{DBD}$ half-life, corresponding to a limit of $0.2-1.1 \mathrm{eV}$ on $\left\langle m_{\nu}\right\rangle$, represents the best limit after those reached by Ge diodes experiments (see table 3 ).

Half-way with next-generation experiments, NEMO III [45] is a passive source detector located in the Frejus underground laboratory at a depth of $\sim 4800$ m.w.e. It consists of a tracking (wire chambers filled with an ethyl-alcohol mixture, operated in the Geiger mode) and a calorimetric (1940 plastic scintillators) system operated in a 30 gauss magnetic field. A well designed source system allows the simultaneous analysis of up to $10 \mathrm{~kg}$ of different $0 \nu \mathrm{DBD}$ active isotopes. Despite a relatively modest energy resolution ( $11 \%$ FWHM at $3 \mathrm{MeV})$, implying a non-negligible background contribution from $2 \nu \mathrm{DBD}$, they achieved very good results on the study of the $2 \nu \mathrm{DBD}$ spectra of several $\beta \beta$ emitters $\left({ }^{82} \mathrm{Se},{ }^{96} \mathrm{Zr},{ }^{116} \mathrm{Cd},{ }^{150} \mathrm{Nd}\right)$. However, regarding the $0 \nu \mathrm{DBD}$ a good result was obtained with ${ }^{100}$ Mo (see table 3 ).

In January 2002, a few members of the HM collaboration claimed evidence for ${ }^{76} \mathrm{Ge} 0 \nu \mathrm{DBD}$ [46] with $T_{1 / 2}^{0 \nu}=0.8-18.3 \times 10^{25}$ y (best value $T_{1 / 2}^{0 \nu}=1.5 \times 10^{25} \mathrm{y}$ ) corresponding to a $\left\langle m_{\nu}\right\rangle$ range of $0.11-0.56 \mathrm{eV}$ (best value $0.39 \mathrm{eV})$. This claim is based on the identification of tiny peaks close to the $0 \nu \mathrm{DBD}$ region of ${ }^{76} \mathrm{Ge}$, one of them at the energy of the $Q$-value of the DBD. However this announcement raised immediate scepticism [47]. Several reanalyses of the data were published by the claim's authors $[48,49,50,51]$, while other authors [52,53,54] still criticize the claim. Probably a definite answer to the correctness of the claim will be given only by the very sensitive next-generation $0 \nu \mathrm{DBD}$ projects.

\section{Towards second-generation experiments}

We have seen that the field of $0 \nu \mathrm{DBD}$ searches is very active. The goal of the future experiments is to reach sensitivities capable of probing the inverted mass hierarchy, i.e. sensitivities in the decay time of the order of $\sim 10^{26}-10^{27}$ years. There are several possibilities in order to improve the sensitivities of the experiments. It is up to the experimentalist to choose the philosophy of the experiment and, consequently, to select the detector characteristics, privileging some properties with respect to others, having clearly in mind the final sensitivity of the set-up to half-life and, consequently, to $\left\langle m_{\nu}\right\rangle$. The question is: how to improve the experiments? Most of the criteria that need to be considered when optimizing the design of a new $0 \nu \mathrm{DBD}$ experiment follow directly from eq. (10) combined with eq. (3); the sensitivity at $68 \%$ CL on the neutrino mass, $F_{0 \nu}$, can be written as

$$
F_{0 \nu}=\frac{m_{e}}{\sqrt{\ln 2 N_{A}}} \frac{1}{\sqrt{G^{0 \nu}\left|M^{0 \nu}\right|^{2}}} \sqrt{\frac{A}{i . a . \epsilon x}} \sqrt[4]{\frac{B \Delta E}{M t}} .
$$

\subsection{Exposure time}

The first consideration is that an "improvement" could be simply reached with the present experiments just by measuring for longer time; from eq. (11) we have

$$
\left.\left\langle m_{\nu}\right\rangle\right|_{t \rightarrow \infty}=\left\langle m_{\nu}\right\rangle_{\Delta T}\left(\frac{\Delta T}{\Delta T+t \rightarrow \infty}\right)^{1 / 4},
$$

where $\Delta T$ is the measurement lifetime that has allowed the present limit on $\left\langle m_{\nu}\right\rangle_{\Delta T}$. Now, almost all the experiments (except the CUORICINO and NEMO III experiments, that have, respectively, a lifetime of $\Delta T \sim 4$ months and of $\Delta T \sim 13$ months) have $\sim \Delta T \geq 2 \mathrm{y}$. This implies that to have an improvement of only a factor 2 in the neutrino mass one has to measure $\sim 30$ years! From this consideration it is also clear that, generally, DBD experiments have a, somewhat, "short" life.

\subsection{Mass}

The mass is one of the "parameters" with which one needs to deal. Regarding this point one has to consider also other variables, i.e. the isotopic abundance. From table 3 we see that all the interesting $\beta \beta$ emitters (except ${ }^{130} \mathrm{Te}$ ) have isotopic abundances of the order of $5-10 \%$. Let us consider, for example, two experiments with the same environmental natural background and with the same mass; let us suppose that one has the $\beta \beta$ emitter with natural i.a. (for example $5 \%$ ) while the second has a $90 \%$ enrichment; then, from eq. (11), it turns out that the sensitivity of the enriched experiment is a factor $\sqrt{90 / 5}=4.2$ better with respect to the other. This result can be seen also from another point of view: the time needed for the 
non-enriched experiment to reach the sensitivity of the enriched one will be $(90 / 5)^{2}=324$ times larger! This point is crucial and, in fact, all the most sensitive experiments carried out in the past (except the ones based on ${ }^{130} \mathrm{Te}$ ) used enriched materials. This holds (see next section) also for the next planned experiments. It is also clear that enrichment is extremely expensive and raises tremendously the cost of an experiment.

Another crucial point comes directly from eq. (9). From table 1 it turns out that the decay time in order to reach a sensitivity on neutrino mass of the order of $10 \mathrm{meV}$ (the goal of the most ambitious next-generation experiments) is of the order of $10^{28} \mathrm{y}$. Thus, assuming a measurement of three years, supposing to have zero background (it is clear that this is practically impossible) and considering at least three events for the $0 \nu \mathrm{DBD}$ discovery, then we need to have at least $N_{\beta \beta}^{*}=10^{28} / \ln 2 \beta \beta$ nuclei, or $\approx 24 \cdot 10^{3}$ moles. It is therefore clear that the mass scale for the second-generation experiments is of the order of a ton. It is also clear that if no signal is detected with the next experiments it will be necessary to cover the normal hierarchy region, i.e. a few $\mathrm{meV}$. Using the same argument it turns out that such third-generation experiments (if any) should be able to reach decay times of the order of $\sim 10^{30} \mathrm{y}$ with a mass of $\sim 100$ ton.

\subsection{Background}

The background is a very delicate point and represent the main task of DBD experiments. There are several background sources that need to be taken into account. Cosmic-ray-induced background can, in principle, be made negligible operating deep underground and using, furthermore, active shielding to discard events correlated with the passage of a nearby muon. Neutron induced background (mainly $(n, \gamma)$ reactions) can also be lowered to a negligible contribution by shielding the experimental setup with suitable moderators/neutron-catchers. It turns out, therefore, that the main source of background is the one arising from the natural radioactivity (mainly ${ }^{232} \mathrm{Th}$ and ${ }^{238} \mathrm{U}$ ) overall present in traces in all kind of materials. Therefore the main work in order to build an experiment is the screening of materials used for the setup. The present techniques are based on High-Purity Ge diodes, neutron activation analysis (for solid and liquid samples), and High-Resolution Inductively Coupled Plasma Mass Spectrometry (HR-ICPMS) for liquid samples. The best limits that can be obtained with the first two techniques are around $10^{-11_{-}}-10^{-12} \mathrm{~g} / \mathrm{g}$ (grams of contaminant/grams of sample) while $10^{-13} \mathrm{~g} / \mathrm{g}$ can be reached on liquid samples. Both techniques are extremely delicate and, moreover, extremely long: for Ge measurement the time needed in order to reach such limits is of the order of several months.

Having screened all the samples of materials to be used for the experiment, one has to assemble the setup. Assuming that all the batches (also the ones that underwent mechanical machining) are as clean as the screened samples, extreme care has to be used in order to avoid possible recontamination. Clean room operation is absolutely needed and operation in "synthetic" air (without Radon) or under clean atmosphere (pure nitrogen) is necessary. The background levels that need to be reached for the next experiments (see sect. 8) require reduction of the order of 10-100 (this means $10-1 \mathrm{c} /(\mathrm{keV} \cdot \mathrm{ton} \cdot \mathrm{y}))$ with respect to the previous "pilot" experiments. These levels of radiopurity cannot be directly tested with the standard techniques [55], so that the only way to measure them will be the experiment itself. Many physicists consider this somehow as "bet".

Strictly connected with the background is also the choice of the $\beta \beta$ emitter. Apart some extremely rare high energy $\gamma$ 's from the ${ }^{238} \mathrm{U}$ chain, the highest natural $\gamma$ line arising from natural radioactivity is the $2615 \mathrm{keV}$ line of ${ }^{208} \mathrm{Tl}$ (from ${ }^{232} \mathrm{Th}$ chain). It would be, therefore, extremely useful to choose a $\beta \beta$ emitter with a $\mathrm{Q}_{\beta \beta}$ value above this energy.

\subsection{Energy resolution}

Energy resolution will be the key point for some future experiments. Apart from the obvious role played in eq. (11), there is another fundamental aspect that has to be addressed. Assuming also the ability to reduce all the background sources, there is an intrinsic, unavoidable, undistinguishable "background": the $2 \nu \mathrm{DBD}$. This source of background did not play a reasonable role in the present and past experiments due to the "low" sensitivity reached. As pointed out in [56] the fraction $F$ of $2 \nu \mathrm{DBD}$ events that are contained in the $\Delta E^{\mathrm{FWHM}}$ energy window centred at the $Q_{\beta \beta}$ value is given by

$$
F \approx 7 \frac{Q_{\beta \beta} \delta^{6}}{m_{e}}, \quad \delta=\frac{\Delta E^{\mathrm{FWHM}}}{Q_{\beta \beta}} .
$$

An expression for the $0 \nu \mathrm{DBD}$ signal $(S)$ to the $2 \nu \mathrm{DBD}$ background $(B)$ ratio can be written

$$
\frac{S}{B} \approx \frac{m_{e}}{7 Q_{\beta \beta} \delta^{6}} \frac{T_{1 / 2}^{2 \nu}}{T_{1 / 2}^{0 \nu}} .
$$

For example, looking at table 3 , taking $T_{1 / 2}^{2 \nu} \approx 10^{20} \mathrm{y}$ and $Q_{\beta \beta} \approx 3 \mathrm{MeV}$, in order to reach a sensitivity on $T_{1 / 2}^{0 \nu}$ of the order of $10^{27}\left(10^{28}\right)$ y with $S / B=1$ the energy resolution should be less than $3.7(2.5) \%$ FWHM at the $Q_{\beta \beta}$ value. This energy resolution is extremely challenging for all detectors except $\mathrm{Ge}$ diodes and bolometers that have, normally, energy resolutions of the order of $0.2-0.4 \%$ FWHM. For the sake of completeness it has to be noted that the $S / B$ ratio can be slightly enhanced by choosing an asymmetric analysis window defined as $Q_{\beta \beta}<E<Q_{\beta \beta}+\Delta E^{\mathrm{FWHM}}$.

\section{Future experiments}

So far, the best results have been obtained by exploiting the calorimetric approach (active source detectors) which characterizes therefore most of the future proposed 
Table 4. Expected sensitivities of future projects. Last column evaluated from table 1 .

\begin{tabular}{ccccc}
\hline Experiment & Isotope & $\begin{array}{c}\text { kMoles } \\
(\beta \beta)\end{array}$ & $\begin{array}{c}T_{1 / 2}^{0 \nu} \\
\left(10^{26} \mathrm{y}\right)\end{array}$ & $\begin{array}{c}\left\langle m_{\nu}\right\rangle \\
(\mathrm{meV})\end{array}$ \\
\hline CUORE [57] & ${ }^{130} \mathrm{Te}$ & 1.6 & 7 & $(46-91)$ \\
EXO [58] & ${ }^{136} \mathrm{Xe}$ & 48 & 130 & $(5-30)$ \\
GERDA [59] & ${ }^{76} \mathrm{Ge}$ & 0.5 & 2 & $(105-300)$ \\
MAJORANA [60] & ${ }^{76} \mathrm{Ge}$ & 5.6 & 40 & $(24-66)$ \\
MOON III [61] & ${ }^{100} \mathrm{Mo}$ & 8.5 & 30 & $(15-36)$ \\
XMASS [62] & ${ }^{136} \mathrm{Xe}$ & 6.1 & 30 & $(9-63)$ \\
DCBA [63] & ${ }^{150} \mathrm{Nd}$ & 2.7 & 1 & $(16-55)$ \\
SUPERNEMO [64] & ${ }^{82} \mathrm{Se}$ & 1.1 & 2 & $(55-170)$ \\
CAMEO III [65] & ${ }^{116} \mathrm{Cd}$ & 2.7 & 10 & $(20-68)$ \\
CANDLES IV [66] & ${ }^{48} \mathrm{Ca}$ & 0.6 & 30 & $(55)$ \\
\hline
\end{tabular}

projects. Actually, a series of new proposals has been boosted by the recent renewed interest in $0 \nu \mathrm{DBD}$ following neutrino oscillation results. It is not so easy to classify them:

1. High energy resolution calorimetric experiments based on already consolidated techniques with improvements in background suppression/rejection (e.g. CUORE, GERDA, MAJORANA).

2. Calorimetric experiments based on consolidated techniques of scintillation light detection (CANDLES, CAMEO).

3. Calorimetric experiments with or without background identification techniques based on non-standard techniques that require further R\&D (EXO, XMASS).

4. "Passive" experiments based on standard techniques that requires R\&D (SUPERNEMO, MOON, DCBA).

Expected sensitivities on $T_{1 / 2}^{0 \nu}$ of the proposed projects are compared in table 4 . The sensitivities on $\left\langle m_{\nu}\right\rangle$ are evaluated using table 1 . In many cases technical feasibility tests are requested, but the crucial issue will be the capability of each project to pursue the expected background suppression.

Many proposals have been recently suggested. However most of them are not officially approved or require further R\&D to actually prove the feasibility. A complete report can be found in $[67,68]$.

GERDA [59] (GERmanium Detector Array) is the only completely approved and funded experiment. It will be carried on in the INFN Gran Sasso National Laboratories. It is based on the technique already suggested by the HM Collaboration [69]: "naked" Ge diodes will be suspended in the centre of a very large liquid nitrogen container, which will act as a very effective shield. The experiment will consist of two phases: in the first one the same detectors of the HM Collaboration and the IGEX Collaboration will be "naked", removing all the components that are not needed for operating them in liquid nitrogen. The total mass will be $\sim 17 \mathrm{~kg}$ of $86 \%$ enriched ${ }^{76} \mathrm{Ge}$. The collaboration will probe the HM claim within the first 1-2 years. They plan to reach zero background in the $0 \nu \mathrm{DBD}$ region. Therefore, if the result of the HM
Collaboration is true they will expect to confirm it at $5 \sigma$ CL within the first year of operation. The second phase will consist of the addition of new enriched detectors for a total mass of $60 \mathrm{~kg}(0.7 \mathrm{kmol})$. For the second phase they will use background discrimination techniques and they, again, quote zero background. The first phase should start data taking in 2007.

CUORE [57] (Cryogenic Underground Observatory for Rare Events) will be a larger extension of CUORICINO. The experiment is scientifically approved and partially funded. CUORE will consists of 25 CUORICINO-like towers with $9885 \times 5 \times 5 \mathrm{~cm}^{3} \mathrm{TeO}_{2}$ non-enriched crystals. The number of $\beta \beta$ nuclei will be $1.6 \mathrm{kmol}$. The construction of a large custom cryostat, already funded, will start in 2006. Among all the proposed experiments it is the only one that needs a background suppression of "only" a factor 10 with respect to the "pilot" CUORICINO experiment. A factor of two was already achieved in 2004. The expected sensitivity will be $7 \cdot 10^{26} \mathrm{y}$. The experiment should start in 2009.

MAJORANA [60], which involves many of the IGEX collaborators, will consist of an array of 210 isotopically enriched Ge diodes for a total mass of 0.5 tons. As opposed to the GERDA design, the use of a very low activity conventional cryostat (extremely radiopure electroformed $\mathrm{Cu}$ ) able to host simultaneously a large number of diodes is proposed. The driving principle behind the project is a strong reduction of the background by the application of very effective pulse-shape discrimination and the development of special segmented detectors. Despite the very promising $\mathrm{R} \& \mathrm{D}$ developed in the last years, the project is not yet funded.

\section{Conclusions}

A renewed interest in $0 \nu \mathrm{DBD}$ has been stimulated by recent neutrino oscillations results. Neutrinoless DBD is finally recognized as a unique tool to measure neutrino properties (nature, mass scale, intrinsic phases) unavailable for the successful experiments on neutrino oscillations. The attainability of such a goal strongly depends on the true capability of these projects to reach the required background levels in the $0 \nu \mathrm{DBD}$ region. An experimental confirmation of the (sometimes optimistic) background predictions of the various projects (even if extrapolated from the results of lower-scale successful experiments) is therefore worthwhile and the construction of preliminary test setups is absolutely needed. The ultra-low background understanding is a very complicated issue that needs careful investigations and experimental confirmation. The recently claimed evidence for a $0 \nu$ DBD signal in the HM data seems still too weak but will be verified by the future experiments.

A strong effort to improve the NME evaluation should be encouraged while stressing the need of experiments addressed to different nuclei. 


\section{References}

1. S.J. Freedman, B. Kayser (Co-chairs of the organizing committee), arXiv:physics/0411216.

2. R.N. Mohapatra, G. Senjanovic, Phys. Rev. D 23, 165 (1981).

3. B. Kayser, The Physics of Massive Neutrinos (World Scientific, Singapore, 1989).

4. K. Fujikawa, R. Shrock, Phys. Rev. Lett. 45, 963 (1980).

5. J. Kaulard et al., Phys. Lett. B 422, 334 (1998).

6. B. Pontecorvo, Zh. Eksp. Teor. Fiz. 33, 549 (1957); 34, 247 (1958); Z. Maki, M. Nakagawa, S. Sakata, Prog. Theor. Phys. 28, 870 (1962); B. Pontecorvo, Zh. Eksp. Teor. Fiz. 53, 1717 (1967).

7. Ling-Lie Chau, Wai-Yee Keung, Phys. Rev. Lett. 53, 1802 (1984).

8. P.J. Siemens, A.S. Jensen, Elements of Nuclei (AddisonWesley, 1987).

9. S.R. Elliot et al., Phys. Rev. Lett. 59, 2020 (1987).

10. V.I. Tretyak, Yu.G. Zdesenko, At. Data Nucl. Data Tables 80, 83 (2002).

11. H. Ejiri, Prog. Part. Nucl. Phys. B 48, 185 (2002).

12. W.H. Furry, Phys. Rev. 56, 1184 (1939).

13. V.A. Rodin, A. Faessler, F. Simkovic, arXiv:nuclth/0503063 (2005).

14. E. Caurier et al., Nucl. Phys. A 654, 973 (1999).

15. J. Engel et al., Phys. Rev. C 37, 731 (1988).

16. A. Staudt et al., Europhys. Lett 13, 31 (1990).

17. G. Pantis et al., Phys. Rev. C 53, 695 (1996).

18. O. Civitarese, J. Suhonen, Nucl. Phys. A 729, 867 (2003).

19. A. Strumia, F. Vissani, arXiv:hep-ph/0503246.

20. J. Suhonen, Nucl. Phys. B (Proc. Suppl.) 143, 240 (2005).

21. J. Suhonen, Phys. Lett. B 607, 87 (2005).

22. H. Ejiri, Phys. Rep. 338, 265 (2000).

23. B.T. Cleveland et al., Astrophys. J. 496, 505 (1998).

24. C. Cattadori, N. Ferrari, L. Pandola, Nucl. Phys. B (Proc. Suppl.) 143, 3 (2005).

25. Super-Kamiokande Collaboration, hep-ex/0205075.

26. SNO Collaboration, nucl-ex/0502021.

27. KamLAND Collaboration, hep-ex/0406035.

28. S. Goswami, A. Bandyopadhyay, S. Choubey, Nucl. Phys. B (Proc. Suppl.) 143, 121 (2005).

29. A. Lewis, S. Bridle Phys. Rev. D 66, 103511 (2002); M. Tegmark et al., Phys. Rev. D 69, 103501 (2004); P. Crotty, J. Lesgourgues, S. Pastor, Phys. Rev. D 69, 123007 (2004).

30. V.B. Brudanin et al., Phys. Lett. B 495, 63 (2000).

31. HM Collaboration (H.V. Klapdor-Kleingrothaus et al.), Eur. Phys. J. A 12, 147 (2001).

32. C.E. Aalseth et al., Nucl. Phys. B 48, 223 (1996).

33. C.E. Aalseth et al., Phys. Rev. C 59, 2108 (1999).

34. IGEX Collaboration (C.E. Aalseth et al.), hepex/0202026.
35. R. Arnold et al., Phys. Rev. Lett. 95, 182302 (2005).

36. F. Danevich et al., Phys. Rev. C 68, 035501 (2003).

37. C. Arnaboldi et al., Phys. Lett. B 557, 167 (2003).

38. C. Arnaboldi et al., Phys. Rev. Lett. 95, 142501 (2005).

39. R. Bernabei et al., Phys. Lett. B 546, 23 (2002).

40. A. De Silva et al., Phys. Rev. C 56, 2451 (1997).

41. T. Kirsten, H. Richter, E. Jessberger, Phys. Rev. Lett. 50, 474 (1983); A.L. Turkevich et al., Phys. Rev. Lett. 67, 3211 (1991).

42. E. Fiorini, T. Niinikoski, Nucl. Instrum. Methods 224, 83 (1984).

43. A. Alessandrello et al., Nucl. Instrum. Methods A 440, 397 (1998).

44. C. Arnaboldi et al., Nucl. Instrum. Methods A 554, 300 (2005).

45. R. Arnold et al., Nucl. Instrum. Methods A 536, 79 (2005).

46. H.V. Klapdor-Kleingrothaus, A. Dietz, H.V. Harney, I.V. Krivosheina, Mod. Phys. Lett. A 16, 2409 (2001).

47. C.E. Aalseth et al., Mod. Phys. Lett A 17, 1475 (2002).

48. H.V. Klapdor-Kleingrothaus et al., arXiv:hep-ph/0205228.

49. H.V. Klapdor-Kleingrothaus et al., Nucl. Instrum. Methods A 510, 281 (2003).

50. H.V. Klapdor-Kleingrothaus et al., Nucl. Instrum. Methods A 511, 335 (2003).

51. H.V. Klapdor-Kleingrothaus et al., Phys. Lett. B 578, 54 (2004).

52. Yu.G. Zdesenko et al., Phys. Lett. B 546, 206 (2002).

53. F. Feruglio et al., Nucl. Phys. B 637, 345 (2002).

54. A. Giuliani, Nucl. Phys. B (Proc. Suppl.) 138, 267 (2005).

55. G. Heusser, AIP Conf. Proc. 785, 39 (2005).

56. S. Elliott, P. Vogel, Annu. Rev. Nucl. Part. Sci. 52, 115 (2002).

57. C. Arnaboldi et al., Nucl. Instrum. Methods A 518, 775 (2004).

58. M. Danilov et al., Phys. Lett. B 480, 12 (2000).

59. GERDA Collaboration (Hardy Simgen), Nucl. Phys. B (Proc. Suppl.) 143, 567 (2005).

60. The Majorana White Paper, 2003 Preprint, nuclex/0311013.

61. H. Ejiri et al., Phys. Rev. Lett. 85, 2917 (2000).

62. S. Moriyama et al., Proceedings of the XENON01 Workshop, December 2001, Tokyo, Japan (2001).

63. Y. Kato et al., Nucl. Instrum. Methods A 498, 430 (2003).

64. NEMO Collaboration (F. Piquemal), talk presented at the IN2P3 scientific committee, 21 March 2005.

65. G. Bellini et al., Eur. Phys. J. C 19, 43 (2001).

66. T. Kishimoto et al., Osaka University 2001 Annual Report.

67. F.T. Avignone III et al., New J. Phys. 7, 6 (2005).

68. S.R. Elliot, J. Engel, J. Phys. G 30, R183 (2004).

69. H.V. Klapdor-Kleingrothaus, hep-ph/0103074. 